\title{
Texture Analyses and Microstructural Evolution in Irradiated Monolithic U-Mo Nuclear Fuel
}

Fidelma Di Lemma, Jatuporn Burns, Alexander Winston, James Madden, Jan-Fong Jue and Dennis Keiser

Idaho National Laboratory, Idaho Falls, Idaho, United States

Great effort worldwide has been invested in replacing highly enriched uranium (HEU) fuel with lowenriched uranium (LEU) fuel in nuclear plants, in the frame of safeguarding nuclear material and promoting the proliferation resistance [1]. A monolithic U-Mo fuel type is being developed at Idaho National Laboratory for this purpose. This study focuses on the characterization of fresh and irradiated monolithic U-Mo fuel specimens to understand the effect of irradiation on the initial texture. Understanding, controlling and monitoring texture evolution under irradiation is important to evaluate material properties, as texture can affect up them up to $20-50 \%$ (e.g. Young's modulus, Poisson's ratio, strength ductility, toughness, thermal expansion, and magnetic-electrical properties) [2].

Samples texture was analyses via Electron Back Scattered Diffraction (EBSD), using the Quanta 3D FEG Dual Beam equipped with the EDAX Hikari EBSD. Sample preparation was a challenging and important task of this work as EBSD patterns are generated within a small interaction volume located at a depth of less than 50-100 $\mathrm{nm}$. Thus, the data quality is extremely sensitive to the integrity of the crystallographic lattice order at the surface of the sample and to oxidation [2]. While conventional sample preparation methods are widely used in materials science, they often do not lead to quality electron backscattered patterns needed for characterization of the nuclear materials [3]. Due to challenges associated with handling radiological specimens, rapid oxidation of the specimens, and "artifacts" (high number of defects, precipitates and/or porosities) [3-7]. Thus, the Focused Ion Beam (FIB) glazing technique [1,4,6] has been used and optimized for EBSD collection for this fuel type. High Voltage $(30 \mathrm{kV})$ and low current (7nA) was used during FIB cleaning to minimize beam damaged to the sample.

From the EBSD analyses not only texture information, but also phases and grain size data was obtained. The fresh fuel samples showed large grains 5-20 $\mu \mathrm{m}$ (Figure 1). Rolling texture could be observed along

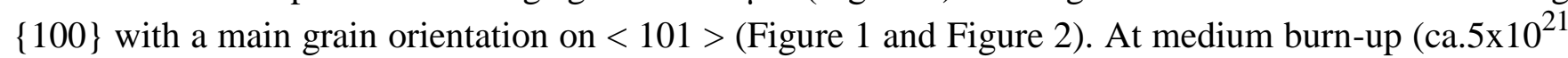
fission/cc) the samples grain structure was modified. The sample developed a bimodal distribution, where some of the original grains are maintained (ca.10 $\mu \mathrm{m}$ diameter) and smaller grains (200-300 $\mathrm{nm}$ ) are formed. The process involved with the observed grain subdivision ("grain refinement") under irradiation has been widely discussed [4], not only for U-Mo system but also for other fuel types. The mechanism for grain subdivision has been described either as polygonization or recrystallization [4]. Our work shows that most of grain boundaries formed around the refined grains are low angle, in line with the polygonizations mechanism. When evaluating the texture for the irradiated sample, it can be observed that some of the initial texture is retained in the bigger grains (Figure 2). The refined grains, on the other hand, show a new texture. Detailed investigation is ongoing to evaluate if this effect is an artifact induced by erroneous indexing or it is related to the subdivision mechanism.

Acknowledgement The work presented is part of the United States High-Performance Research Reactor (USHPRR) program under the United States Department of Energy (DOE), National Nuclear Security Administration's (NNSA) NA-23. 

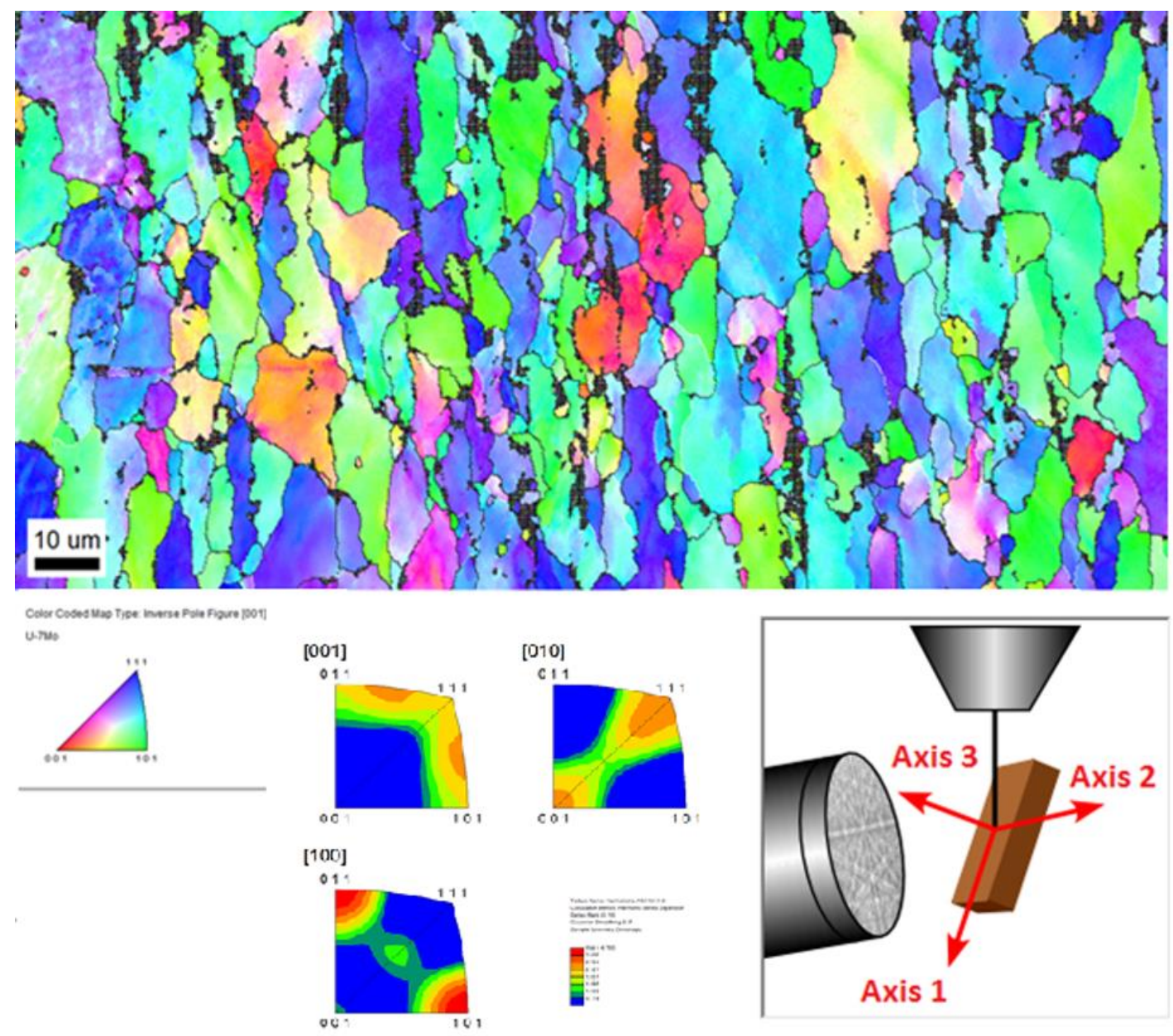

Figure 1. -IPF map on fresh fuel top. Bottom texture analyses.


Figure 2. Pole Figures, Left PF associated with the fresh fuel showing the developed texture (each point represents a grain weight by its dimension). Middle PF for the irradiated sample showing that the bigger grains maintained the texture(each point represent a grain weight by its dimension), while the refined grains (right PF) show a new texture being developed(each point represent an indexed point/pixel). 


\section{References}

[1] A.J. Clarke, et al., J. Nucl. Mater. 465 (2015), 784-792.

[2] Olaf, Randle Introduction to texture analysis 2nd edition 2010

[3] Ajantiwalay T., et al. Micron, 118, March (2019), 1-8.

[4] D. Jadernas, et al., "J. Nucl. Mater. 509 (2018) 1-8.

[5] ILtis X. et al. Journal of Nuclear Materials, 495, November (2017), 249-266.

[6] Aitkaliyeva A., et al. Micron 67 (2014) 65-73.

[7] Nowell M., et al. AMETEK Material Analysis Division. "How I Prepare Samples for EBSD Analysis". 